\title{
Theoretical asteroseismology of solar-like oscillations
}

\author{
G. Houdek
}

Institute of Astronomy, University of Cambridge, Cambridge CB30HA, UK

\begin{abstract}
Having a rich acoustic oscillation spectrum makes solar-like stars particularly interesting for studying fluid-dynamical aspects of the stellar interior. I present some of the recent progress in formulating the physical processes that drive the acoustic oscillations to the observed amplitudes via their coupling with the turbulent velocity field in the outer convectively unstable stellar layers. I shall also discuss some asteroseismic diagnostic techniques that allow us to measure some of the gross stellar properties derived from a seismic signature contained in the variation of the large frequency separation of measured low-degree acoustic modes.
\end{abstract}

\section{Introduction}

Solar-type stars possess extended surface convection zones. The observed oscillation modes generally behave as acoustic modes and their frequencies are sensitive predominantly to the sound speed in the stellar interior. It appears that all possible oscillation modes are intrinsically stable. They are excited stochastically by the strong emission of acoustic noise by the turbulent velocity field in the upper convectively unstable layers of the star. The excitation occurs in a broad frequency range, giving rise to a rich pulsation spectrum. The amplitudes of the oscillations are small, typically $5 \mathrm{ppm} L_{\odot} / M_{\odot}$ (Kjeldsen \& Bedding 1995), allowing us to describe the pulsations with linear theory.

Only modes of low degree can be observed. The diagnostic properties of this type of mode have been studied extensively in the solar case. From asymptotic theory we find for the cyclic oscillation frequencies $\nu_{n, \ell}$ with radial order $n$ and spherical degree $\ell$ (Gough 1986, see also Tassoul 1980)

$$
\nu_{n, \ell} \simeq(n+\ell / 2+\alpha) \nu_{0}+\varepsilon_{n, \ell},
$$

where $\nu_{0}=\left[2 \int_{0}^{R} \mathrm{~d} r / c\right]^{-1}$ is the inverse of twice the sound travel time between the centre and surface ( $R$ is surface radius), and $\alpha$ is a constant. The value of $\nu_{0}$ can be estimated from taking the average (over $n$ and $\ell$ ) of the so-called large frequency separation $\nu_{n, \ell} \equiv$ $\nu_{n, \ell}-\nu_{n-1, \ell}$. The correction term $\varepsilon_{n, \ell}$ lifts the degeneracy between modes with the same value of $n+\ell / 2$ and leads to the so-called small frequency separation $\delta \nu_{n, \ell} \equiv \nu_{n, \ell}-\nu_{n-1, \ell+2}$. This frequency structure is illustrated in Fig. 1 for a solar spectrum. The small frequency separation is predominantly determined by the acoustic sound speed in the stellar core and hence is sensitive to the chemical composition there and consequently is an indicator for the stellar age (e.g., Gough 2001).

\section{Oscillation amplitudes}

In the Sun and other solar-like oscillators mode stability is governed not only by the perturbations in the radiative fluxes (i.e., via the $\kappa$-mechanism) but also by the perturbations in the turbulent fluxes (heat and momentum). The study of mode stability therefore demands a theory for convection that includes the interaction of the turbulent velocity field with the 


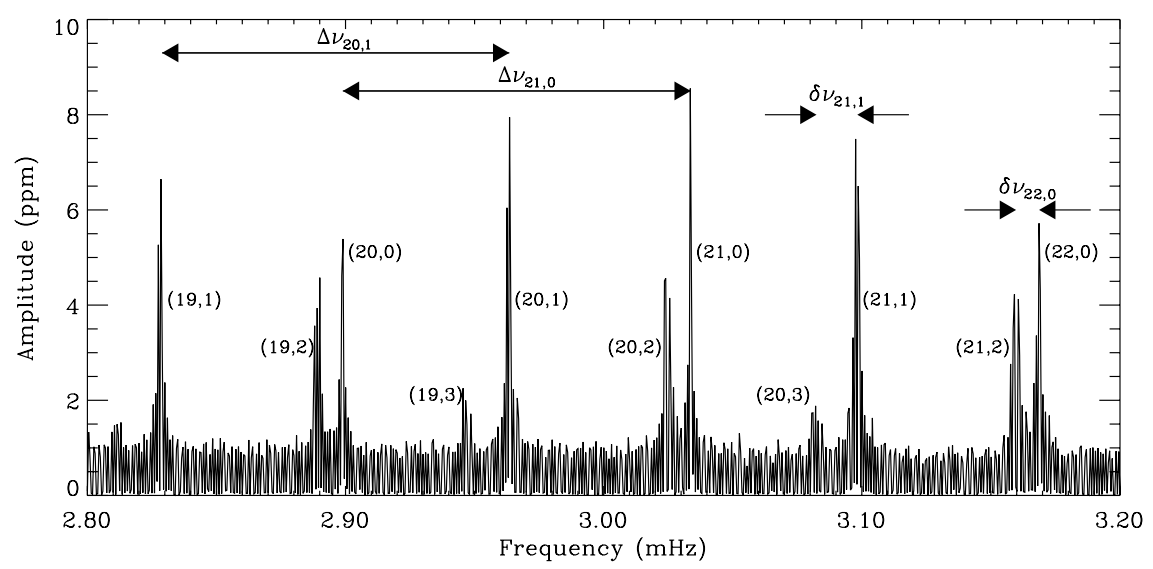

Figure 1: Small section of a solar acoustic power spectrum. The radial order $n$ and spherical degree $\ell$ are indicated in pairs of $(n, \ell)$ for each mode. The large and small frequency separations, $\Delta \nu_{n, \ell}$ and $\delta \nu_{n, \ell}$ are in general functions of $n$ and $\ell$ and can be used to infer the mass and age of a star (adapted from Christensen-Dalsgaard 2001).

pulsation. It appears that in solar-like stars all possible modes of oscillation are stable; thus, if a given oscillation is somehow excited, it will be damped over a finite time, typically of the order of days to months, the inverse of which is the damping rate $\eta$. The power spectrum (Fig. 1) can be described in terms of an ensemble of intrinsically damped, stochastically driven, simple-harmonic oscillators, provided that the background equilibrium state of the star were independent of time. In that case the mode profile is essentially Lorentzian, and the intrinsic damping rates of the modes could then be determined observationally from measurements of the pulsation linewidths. The other fundamental quantity that any full description of mode excitation must model is the energy supply rate, $P$, which is sometimes called the acoustic noise generation rate. The observed velocity signal $v(t)=\mathrm{d} \xi / \mathrm{d} t$ (where $\xi(t)$ is the surface displacement of the damped, stochastically driven, harmonic oscillator) can then be related to the modelled energy supply rate $P$ by taking the Fourier transformation of the harmonic oscillator followed by an integration over frequency to obtain the total mean energy $E$ in a particular pulsation mode with inertia $\mathcal{I}$ (e.g., Chaplin et al. 2005, Houdek 2006). The squared surface rms velocity is then given by

$$
V^{2}:=\frac{E}{\mathcal{I}}=\frac{P}{2 \eta \mathcal{I}}=\frac{1}{2} \eta H
$$

where the height $H$ (in $\mathrm{cm}^{2} \mathrm{~s}^{-2} \mathrm{~Hz}^{-1}$ ) is the maximum of the discrete power, i.e. the integral of power spectral density over a frequency bin. As such, it is not the total integrated power, $V^{2}$, that is observed directly, but rather the power spectral density (Chaplin et al. 2005). The excitation process can be regarded as multipole acoustic radiation (Lighthill 1952). Acoustic radiation by turbulent multipole sources in the context of stellar aerodynamics has been considered by various authors (for a recent review see Houdek 2006). Here we follow the procedure by Chaplin et al. 2005, who derived the following expression for estimating the energy supply rate arising from the fluctuating Reynolds stresses $P_{\mathrm{R}}$ (another contribution comes from the fluctuating gas pressure $P_{\mathrm{g}}$, i.e. the total energy supply rate $P=P_{\mathrm{R}}+P_{\mathrm{g}}$; here we neglect $P_{\mathrm{g}}$ ): 


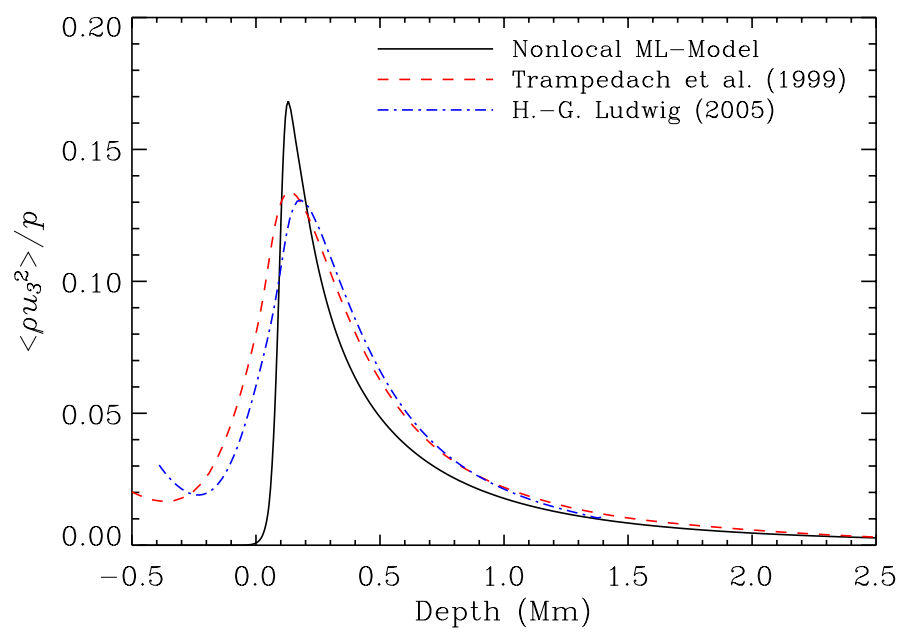

Figure 2: Reynolds stress as a function of the depth variable $z=\mathrm{R}_{\odot}-r$ for various solar models. Results are shown for the non-local mixing-length model (solid curve) and from hydrodynamical simulations by Trampedach et al. (1999, dashed curve) and Ludwig (2005, dot-dashed curve).

$$
P_{\mathrm{R}}=\frac{\pi}{9 \mathcal{I}} \int_{0}^{R} l^{3}\left(\phi \Psi r p_{\mathrm{t}} \frac{\partial \xi_{r}}{\partial r}\right)^{2} \mathcal{S}(r ; \nu) \mathrm{d} r
$$

where $l$ is the mixing length, $p_{\mathrm{t}}$ is the $(r, r)$-component of the Reynolds stress, and $\xi_{r}$ is the normalized radial component of the displacement vector. The spectral function $\mathcal{S}$ accounts for contributions to $P$ from the small-scale turbulence. The parameter $\Psi=[2 \Phi / 3(\Phi-1)]^{1 / 2}$ is unity for isotropic turbulence (Chaplin et al. 2005) and is obtained from a consistent kinematic transformation of the turbulent velocity correlation $\langle\mathbf{u u}\rangle$ (angular brackets denote an ensemble average) in the Boussinesq-quasi-normal approximation, where $\Phi=\langle\mathbf{u} \cdot \mathbf{u}\rangle /\left\langle u_{3}^{2}\right\rangle$ describes the anisotropy of the turbulent velocity field $\mathbf{u}=\left(u_{1}, u_{2}, u_{3}\right)$.

The relative $(r, r)$-component of the Reynolds stress $p_{\mathrm{t}} / p=\left\langle\rho u_{3}^{2}\right\rangle / p(\rho$ is density and $p$ is the total pressure) is compared with hydrodynamical simulations in Fig. 2 . The Reynolds stress of the non-local mixing-length model shows a narrow peak near the depth $z \simeq 120 \mathrm{~km}$ and falls off more rapidly with $z$ than the results from both hydrodynamical simulations. This contributes to make the energy supply rate for the mixing-length model smaller than that from the hydrodynamical simulations, and consequently the modelled heights $H$ need to be scaled with a scaling factor $>1$ in order to reproduce the observed values of the mode peak heights (Chaplin et al. 2005). With a model for $P$ and estimates for $\eta$ from nonadiabatic pulsation calculations the oscillation amplitude $V$ is obtained from Eq. (2).

Fairly accurate measurements of solar-like oscillation amplitudes in other stars are available today from ground based observations (see Bedding \& Kjeldsen 2007). Results for models of $\alpha$ Cen A and for the sub-giant $\xi$ Hydrae are illustrated in Fig. 3. Bedding et al. (2004) reported mode lifetimes for $\alpha$ Cen $\mathrm{A}$ between 1-2 days which are in reasonable agreement with the theoretical estimates of about 1.7 days for the most prominent modes (the mode lifetime $\tau=\eta^{-1}$; see lower left panel of Fig. 3). For $\xi$ Hydrae, however, the theoretical mode lifetimes of the most prominent modes are $\tau \simeq 17$ days which are in stark contrast to the measured values of about 2-3 days by Stello et al. (2006), yet the estimated velocity amplitudes are in almost perfect agreement with the observations by Frandsen et al. (2002). 

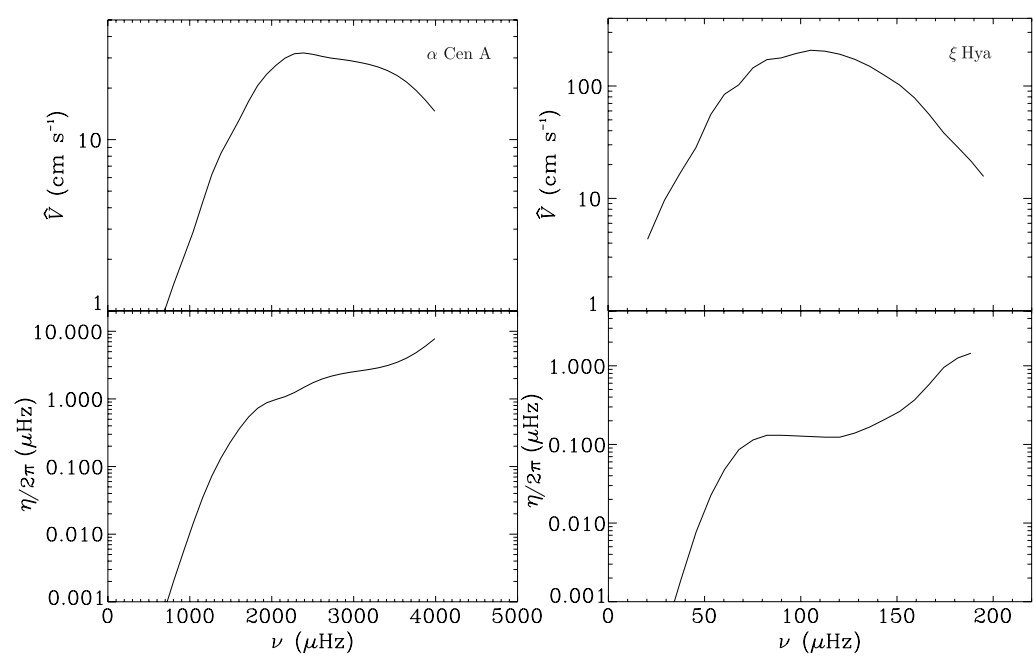

Figure 3: Predicted apparent velocity amplitudes (defined to be $\sqrt{2}$ times the rms value $V$, top) and damping rates (bottom) of radial acoustic modes for models of $\alpha$ Cen A (left) and $\xi$ Hydrae (right).

The predicted maximum velocity amplitudes for various solar-like stars are compared in Fig. 4 with recently performed observations. For the cooler stars the theoretical results are in reasonable agreement with the observations. For the rather hotter star Procyon, however, the theoretical velocity amplitudes are severely overestimated. The dotted line is the scaling law by Kjeldsen \& Bedding (1995), and the dashed line is the scaling relation reported by Samadi et al. (2005) using the convective velocity profiles from numerical simulations (Stein \& Nordlund 2001), and the theoretical damping rates from Houdek et al. (1999). For hotter stars they find better agreement with observations. It is, however, interesting to note that the numerical simulations by Stein et al. (2004) show for hotter stars partial cancellation between the two excitation sources, $P_{\mathrm{R}}$ and $P_{\mathrm{g}}$, arising from the fluctuating Reynolds stresses and gas pressure (buoyancy force) respectively. On average, this results in a total energy supply rate that is smaller by a factor of about two than the energy supply rate from the turbulent pressure fluctuations alone. One is therefore tempted to argue that the overestimated values of the modelled energy supply rate $P$ in Procyon could be partially attributed to having neglected the gas pressure fluctuations in Eq. (2) and in particular its cancellation with the turbulent pressure fluctuations. By adopting the simulated results by Stein et al. (2004) of the energy supply rate $P$ for Procyon the velocity amplitude is reduced by a factor of about $\sqrt{2}$ (indicated by the dot-dashed vertical line in Fig. 4). This suggests, according to Eq. (2), that the remaining factor of about 1.8 , which is necessary to make the estimated velocity amplitude agree with the observed value (dotted vertical line), can be predominantly attributed to the underestimation of the linear damping rates $\eta$ (see Houdek 2006).

\section{The signature of helium ionization}

Abrupt variation in the stratification of a star (relative to the scale of the inverse radial wavenumber of a seismic mode of oscillation), such as that resulting from the (smooth, albeit acoustically relatively abrupt) depression in the first adiabatic exponent $\gamma=(\partial \ln p / \partial \ln \rho)_{s}$ caused by the ionization of helium, where $p, \rho$ and $s$ are pressure, density and specific entropy, 


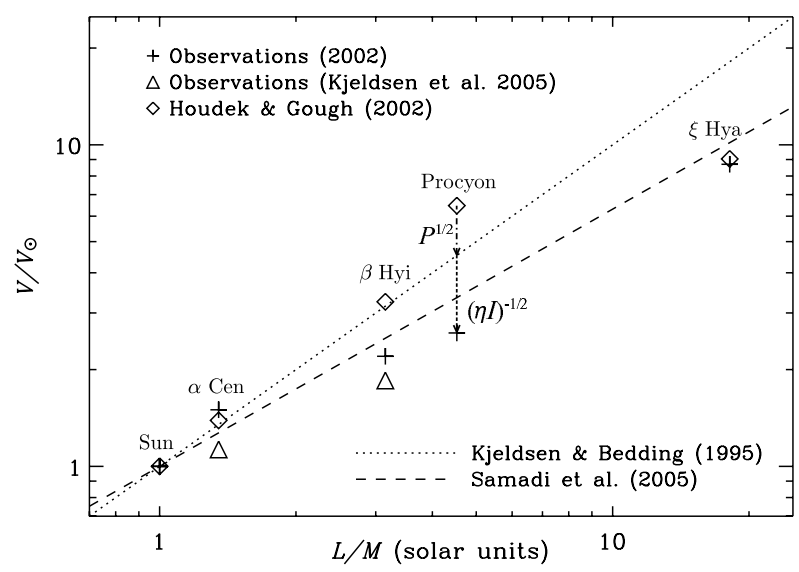

Figure 4: Predicted velocity amplitudes (in solar units) as function of light-to-mass ratio for stochastically excited oscillations in other stars. Observations from several authors are plotted by the plus and triangle symbols. Theoretical estimates are plotted as diamond symbols. The dot-dashed vertical line indicates the reduction of the Procyon amplitude by a factor of $\sqrt{2}$ if the energy supply rate $P$ of Stein et al. (2004) is assumed; the vertical dotted line indicates the remaining factor of about 1.8 by which the theoretical amplitude estimate according to Eq. (2) is still in error with the observations.

or from the sharp transition from radiative to convective heat transport at the base of the convection zone, induces small-amplitude oscillatory components (with respect to frequency) in the spacing of the cyclic eigenfrequencies $\nu_{n, \ell}$ of seismic oscillation and consequently also in $\Delta \nu_{n, \ell}$ and $\delta \nu_{n, \ell}$. We call such abrupt variations an acoustic glitch. One might hope that the variation of the sound speed $c$ induced by helium ionization might enable one to determine from the low-degree eigenfrequencies a measure that is directly related to, perhaps even almost proportional to, the helium abundance, with little contamination from other properties of the structure of the star.

A convenient and easily evaluated measure of the oscillatory component produced by acoustic glitches is the second multiplet-frequency difference with respect to order $n$ amongst modes of like degree $\ell$ :

$$
\Delta_{2} \nu_{n, \ell} \equiv \nu_{n-1, \ell}-2 \nu_{n, \ell}+\nu_{n+1, \ell}
$$

(Gough 1990). Any localized region of rapid variation of either the sound speed $c$ or the density scale height, or a spatial derivative of them, induces an oscillatory component in $\Delta_{2} \nu$ with a 'cyclic frequency' approximately equal to twice the acoustic depth

$$
\tau=\int_{r_{\text {glitch }}}^{R} c^{-1} \mathrm{~d} r
$$

of the glitch, and with an amplitude which depends on the amplitude of the glitch and which decays with $\nu$ once the inverse radial wavenumber of the mode becomes comparable with or less than the radial extent of the glitch.

Various approximate formulae for the oscillatory components that are associated with the helium ionization have been suggested and used, by e.g., Basu et al. (1994, 2004), Monteiro \& Thompson $(1998,2005)$ and Gough (2002), not all of which are derived directly from explicit acoustic glitches. Gough used an analytic function for modelling the dip in the 
first adiabatic exponent. In contrast, Monteiro \& Thompson assumed a triangular form. Basu et al. have adopted a seismic signature for helium ionization that is similar to that arising from a single discontinuity; the artificial discontinuities in the sound speed and its derivatives that this and the triangular representations possess cause the amplitude of the oscillatory signal to decay with frequency too gradually, although that deficiency may not be immediately noticeable within the limited frequency range in which adequate asteroseismic data are or will imminently be available. More recently Houdek \& Gough (2007) proposed a seismic diagnostic in which the variation of $\gamma$ in the helium ionization zone is represented with a pair of Gaussian functions. This correctly results in a decay of the amplitude of the seismic signature with oscillation frequency that is faster than that which the triangular and the single-discontinuity approximations imply, and also takes some account of the two ionization states of helium. Moreover, Houdek \& Gough (2007) incorporated the acoustic cutoff frequency into the variation of the eigenfunction phase with acoustic depth, thereby improving the discrepancy between the seismically inferred depths of the acoustic glitches and that of a corresponding stellar model. In particular these authors suggest the following seismic diagnostic for the oscillatory component associated with helium ionization

$$
\begin{array}{r}
\delta_{\mathrm{osc}} \nu \simeq-\Gamma_{\|} \nu_{0}\left[\nu+\frac{1}{2}(m+1) \nu_{0}\right]\left(\mu \beta \kappa_{l}^{-1} \mathrm{e}^{-8 \pi^{2} \mu^{2} \kappa_{l}^{2} \Delta_{\|}^{2} \nu^{2}} \cos 2 \psi_{l}\right. \\
\left.+\kappa_{\|}^{-1} \mathrm{e}^{-8 \pi^{2} \kappa_{\|}^{2} \Delta_{\| I}^{2} \nu^{2}} \cos 2 \psi_{\|}\right),
\end{array}
$$

in which the dominant glitch term $\delta \gamma / \gamma$ in the helium ionization zone is represented by a pair of (negative) Gaussian functions of acoustic depth $\tau$, with widths $\Delta_{I}$ and $\Delta_{\mathrm{II}}$, whose integrals are $\Gamma_{\mid}$and $\Gamma_{\mid I}$, and which are centred about the acoustic depths $\tau_{\mid}$and $\tau_{\|}$of the first and second ionization zones of helium beneath the seismic surface $r=R$ of the star. The phases $\psi_{\mathrm{I}}=\psi\left(\tilde{\tau}_{\mathrm{I}}\right)$ and $\psi_{\mathrm{II}}=\psi\left(\tilde{\tau}_{\mathrm{I}}\right)$, where $\omega \tilde{\tau}=\omega \tau+\epsilon_{\|}(\omega=2 \pi \nu)$, are evaluated by representing the envelope by a plane-parallel polytrope of index $m=3.5$ and adding a phase constant $\epsilon_{\|}$ to $\omega \tau$ to account for the deviation of the actual envelope from the polytrope:

$$
\psi(\tau)=\omega \tau \kappa-(m+1) \cos ^{-1}\left(\frac{m+1}{\omega \tau}\right)+\frac{\pi}{4}
$$

In Eq. (7), $\kappa_{l}=\kappa\left(\tilde{\tau}_{l}\right)$ etc, with $\kappa(\tau)=\left[1-(m+1)^{2} / 4 \pi^{2} \nu^{2} \tau^{2}\right]^{1 / 2}$. The ratios $\beta=\Gamma_{\mid} \Delta_{\mathrm{II}} / \Gamma_{\mathrm{II}} \Delta_{\mathrm{l}}$, $\mu=\Delta_{\mathrm{I}} / \Delta_{\mathrm{II}}$ and $\tau_{\mathrm{I}} / \tau_{\mathrm{I}}$ hardly vary amongst stellar models whose masses and radii vary by factors of at least five. To complete the description of $\Delta_{2} \nu$ an oscillatory contribution with amplitude $A_{\mathrm{c}}$ (and phase constant $\epsilon_{\mathrm{c}}$ ) from the near discontinuity in the density scale height at the base $\tau_{c}$ of the convection zone is added. It is then straightforward to evaluate the second difference $\Delta_{2} \nu$, to which must be added a smooth term which is represented by a third-degree polynomial in $\nu^{-1}$ :

$$
\Delta_{2, \mathrm{sm}}=\sum_{i=0}^{3} a_{i} \nu^{-i} .
$$

The eleven parameters $\Gamma_{\|}, A_{c}, \Delta_{\|}, \tau_{\| l}, \tau_{c}, \epsilon_{\| l}, \epsilon_{\mathrm{c}}$ and $a_{i}$ are adjusted to fit by least squares the theoretical curve to the second frequency differences of the actual eigenfrequencies of the modes.

The top panel of Fig. 5 shows second differences $\Delta_{2} \nu$ (symbols), defined by Eq. (4), of lowdegree solar frequencies with $\ell=0,1,2$ and 3, obtained from BiSON (Basu et al. 2007). The solid curve is the seismic diagnostic (6)-(8), whose eleven parameters have been adjusted to fit the data by least squares. The values so obtained for the acoustic depth of the centre of the He II ionization zone is $\tau_{\|} \simeq 819 \mathrm{~s}$ and the value for the magnitude of the relative depression 


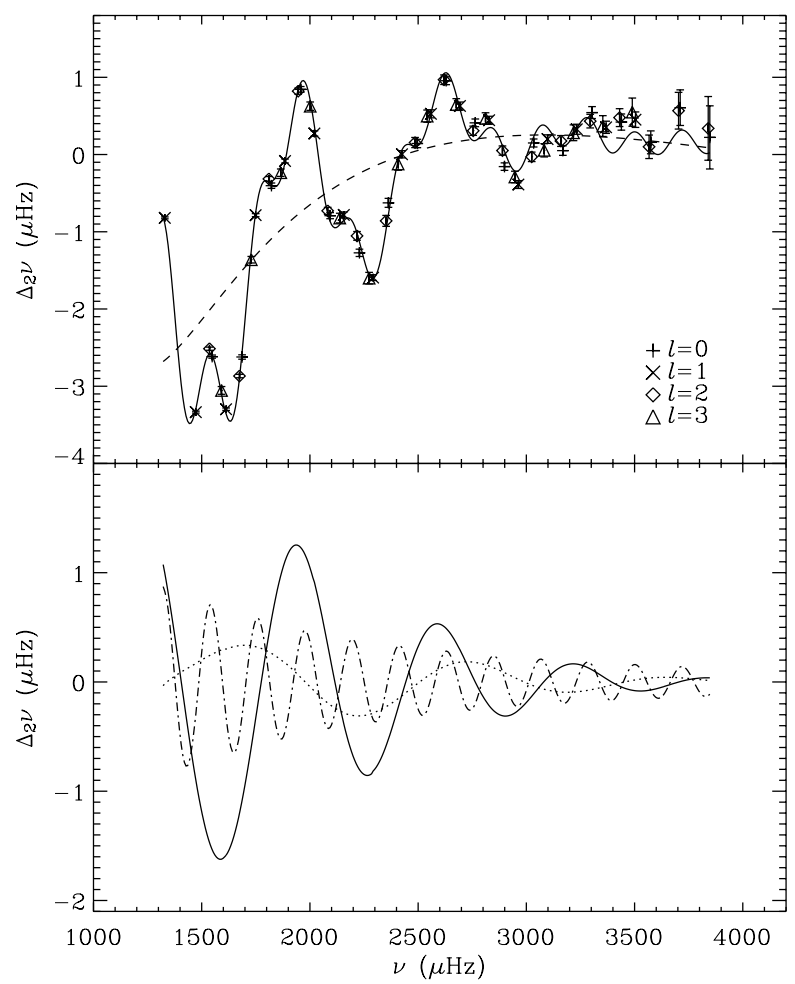

Figure 5: Top: the symbols (with error bars) are second differences $\Delta_{2} \nu$ (Eq. (4)) of low-degree solar frequencies from BiSON (Basu et al. 2007). The solid curve is the diagnostic by Houdek \& Gough (2007) which has been fitted to the data by least squares. The dashed curve represents the smooth contribution of the seismic diagnostic. Bottom: individual (oscillatory) contributions of the seismic diagnostic. The solid curve is the Hell contribution, the dotted curve the Hel contribution and the dot-dashed curve is the contribution from the base of the convection zone.

of $\gamma$ is $-\delta \gamma /\left.\gamma\right|_{\tau_{\|}} \simeq 0.047$. The lower panel of Fig. 5 displays the individual oscillatory contributions from the two ionization stages of helium and from the sharp transition from radiative to convective heat transport at the base of the convection zone.

Such seismic signatures complicate the measurement of the small frequency separation $\delta \nu_{n, \ell}$ which, in general, is used for calibrating stellar models to obtain their ages and initial helium abundances. There is good reason to expect that by considering this oscillatory signature in the calibration process a substantial improvement will be made for determining stellar ages (e.g., Gough 2001).

Acknowledgments. I am grateful to Douglas Gough for many helpful discussions, and to Hans-Günter Ludwig and Regner Trampedach for providing their results for Fig. 2. Support by the Particle Physics and Astronomy Research Council is gratefully acknowledged. 


\section{References}

Basu S., Antia H. M., Narasimha D., 1994, MNRAS, 267, 209

Basu S., Mazumdar A., Antia H. M., Demarque P., 2004, MNRAS, 350, 277

Basu S., Chaplin W. J., Elsworth Y., et al., 2007, ApJ, 655, 660

Bedding T. R., Kjeldsen H., Butler R. P., et al., 2004, ApJ, 614, 380

Bedding T. R., Kjeldsen H., 2007, Comm. Asteroseis., 150, 106

Chaplin W. J., Houdek G., Elsworth Y., et al., 2005, MNRAS, 360, 859

Christensen-Dalsgaard J., 2001, in Favata F., Roxburgh I. W., Galadi D., eds, Stellar Structure and Habitable Planet Finding. ESA SP-485, Noordwijk, p. 25

Frandsen S., Carrier F., Aerts C., et al., 2002, A\&A, 394, L5

Gough D. O., 1986, in Gough D. O., ed., Seismology of the Sun and the distant stars. NATO ASI Ser. C, 169 , p. 125

Gough D. O., 1990, in Osaki Y., Shibahashi H., eds, Progress of Seismology of the Sun and Stars. Lecture Notes in Physics, Vol. 367, Springer Verlag, Heidelberg, p. 283

Gough D. O., 2001, in von Hippel T., Simpson C., Manset N., eds, ASP Conf. Ser. Vol. 245, Astrophysical Ages and Timescales. Astron. Soc. Pac., San Francisco, p. 31

Gough D. O., 2002, in Favata F., Roxburgh I. W., Galadi D., eds, Stellar structure and habitable planet finding. ESA SP-485, Noordwijk, p. 65

Houdek G., Balmforth N. J., Christensen-Dalsgaard J., Gough D. O., 1999, A\&A, 351, 582

Houdek G., 2006, in Fletcher K., ed., SOHO 18/GONG 2006/HelAs I: Beyond the spherical Sun. ESA SP-624, Noordwijk, p. 28.1

Houdek G., Gough D. O., 2002, MNRAS, 336, L65

Houdek G., Gough D. O., 2007, MNRAS, 375, 861

Kjeldsen H., Bedding T. R., 1995, A\&A, 293, 87

Kjeldsen H., Bedding T. R., Butler R. P., et al., 2005, ApJ, 635, 1281

Lighthill M. J. 1952, Proc. Roy. Soc. London, A211, 564

Ludwig H.-G. 2005, personal communication

Monteiro M. J. P. F. G., Thompson M. J., 1998, in Deubner F.-L., Christensen-Dalsgaard J.,

Kurtz D.W., eds, Proc. IAU Symp. 185, New Eyes to see inside the Sun and Stars. Kluwer,

Dordrecht, p. 317

Monteiro M. J. P. F. G., Thompson M. J., 2005, MNRAS, 361, 1187

Samadi R., Goupil M.-J., Alecian E., et al., 2005, J. Astrophys. Astron., 26, 171

Stein R., Nordlund Å., 2001, ApJ, 546, 585

Stein R., Georgobani D., Trampedach R., Ludwig H.-G., Nordlund Å., 2004, Solar Physics, 220, 229

Stello D., Kjeldsen H., Bedding T. R., et al., 2004, Solar Physics, 220, 207

Tassoul M., 1980, ApJS, 43, 469

Trampedach R., Stein R. F., Christensen-Dalsgaard J., Nordlund Å., 1999, in Giménez A., Guinan E. F., Montesinos B., eds, ASP Conf. Ser. Vol. 173, Theory and Tests of Convection in Stellar Structure. Astron. Soc. Pac., San Francisco, p. 233 


\section{DISCUSSION}

Kupka: You mentioned that there may be some physics missing in the prediction of the mode amplitudes. I guess that in any of your models there is nothing that expresses the asymmetries between up- and downflows?

Houdek: It is possible that the effect of acoustic wave scattering on mode damping plays an important role and consequently also on the mode amplitudes. The asymmetries between up- and downflows of the turbulent velocity field could also be important but I don't know to which extent. It would require a convection formulation that goes beyond the Boussinesq approximation.

Kupka: A short comment about Procyon: I believe that you are aware that there is disagreement between the simulations by the Yale group and by H.-G. Ludwig, for example, so we should be more careful with the simulations as compared to the solar case.

Houdek: Yes.

Roxburgh: A word of caution is needed, in the sense that the precision on the solar frequencies is much better than we are ever likely to get for other stars, at least in the near future. Therefore some of the things you are talking about are not realistic when applied to data from satellites as well as from the ground. The other point I would like to make is that you said nothing about the interior structure, but even with data that are worse than for instance the ones from BiSON, with precisions of the order of $0.1-0.2 \mu \mathrm{Hz}$ you can still make inversions to get the interior structure.

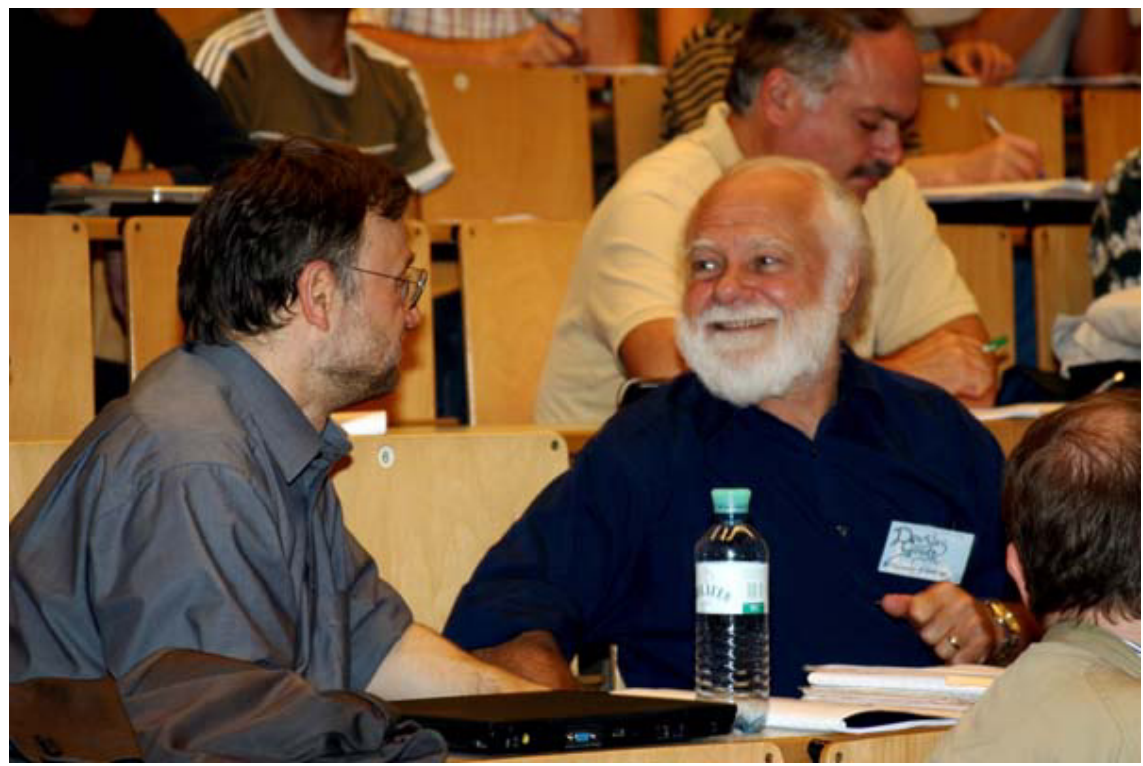

Günter Houdek and Douglas Gough - still discussing solar-like oscillations? 